\title{
Differentiation of neuronal cells using a murine embryonic stem cell-based method
}

\author{
Uthayashanker R. Ezekiel ${ }^{1,2}$ \\ ${ }^{1}$ Department of Clinical Laboratory Science, Doisy College of Health Sciences, Saint Louis University Medical Center, Saint Louis, \\ USA \\ ${ }^{2}$ GeneProTech, Inc., Saint Louis, USA \\ Email: uezekiel@slu.edu
}

Received 24 June 2013; revised 25 July 2013; accepted 10 August 2013

Copyright (C) 2013 Uthayashanker R. Ezekiel. This is an open access article distributed under the Creative Commons Attribution License, which permits unrestricted use, distribution, and reproduction in any medium, provided the original work is properly cited.

\begin{abstract}
The differentiation and screening methodology proposed here is an efficient in vitro system to screen and study effects of small molecules and bioagents and is an alternative to studies that use live animals and embryos. The method is based on engineering a stable murine embryonic stem (ES) cell line expressing lineage-specific promoters that drive selection and reporter genes. Additionally, uniform embryoid bodies (EBs) are used for differentiation studies that allow synchronous differentiation. The reporter and selection marker genes are expressed only in lineages where the promoter is functional. The differentiated cell type can be identified by reporter gene expression and the selection marker can be used for selective enrichment of that particular cell population. The method described here is useful in screening small molecules or bioagents that can differentiate stem cells into particular lineages or cell types. Identified compounds are useful in areas such as stem cell-based regenerative medicine and therapeutics. The method described here has been applied to neuronal cell differentiation.
\end{abstract}

Keywords: Stem Cell; Embryoid Bodies; Differentiation

\section{INTRODUCTION}

Embryonic stem (ES) cells are pluripotent and derived from an early embryonic stage of development. When cultured in the presence of leukocyte inhibitory factor (LIF), ES cells remain undifferentiated. Removal of LIF and regulation of culture conditions lead to ES cell differentiation into specific cell types [1]. Differentiation of ES cells into different cell lineages is of great importance in regenerative medicine. Differentiation into specific cell type(s) and enrichment of the differentiated cell type(s) are critical for therapeutic application purposes. We describe a method to screen small molecules/bioagents that differentiate ES cells to a particular lineage and enrich for a differentiated cell type.

An ES cell line containing a reporter and a selection marker has been engineered with both reporter and selection markers under the control of a specific promoter in the form of a Promoter-Selection-Reporter cassette. The reporter and selection marker genes are expressed only in lineages where the promoter is functional. The differentiated cell type is identified by analysis of reporter gene expression and the importance of the selection marker is selective enrichment of a particular cell population. Several strategies exist to differentiate ES cells into neuronal cell types. Many differentiation strategies involve the generation of embryoid bodies (EBs) that are aggregates of ES cells in suspension [2-5]. Uniform-sized EBs have been shown to have synchronous differentiation potentials; therefore, it is critical to have uniform-size EBs for screening purposes $[6,7]$. In the present study, neuron differentiation has been used as a model system.

Many small molecules exert effects during differentiation. Retinoic acid (RA) is an active metabolite of Vitamin A, differentiates ES cells to neuronal lineages [8] and acts through kinase-dependent pathways in neurogenesis[1,9]. Compounds that exert effects on signaling pathways modulate stem cell differentiation. For example, resveratrol, a natural compound present in grape skins, acts on the extracellular signal-regulated kinase (ERK)-1 pathway in undifferentiated and differentiated neuronlike cells [10]. Neuroprotective effects of Ginkgo biloba extracts have been demonstrated in vitro and in vivo [11, 12]. An analog of Vitamin D having two double bonds in the side chain causes differentiation of MG-63 human osteosarcoma cells into osteoblasts [13]. Rho-associated coiled-coil forming protein kinase (ROCK) inhibitor Y- 
27632 modulates stem cell differentiation [14]. Extracellular signal-regulated kinase (ERK) 1/2 phosphorylation inhibitor U0126 has been used to show ERK pathway involvement in early neuronal differentiation of ES cells [15]. In the present study, small molecules representing natural compounds, kinase inhibitors or other factors promoting neuronal differentiation have been used.

\section{MATERIALS AND METHODS}

\subsection{Construction of Targeting Vector with Neuron-Specific Promoter-Reporter-Selection Cassette}

An 844 bp DNA fragment upstream of the transcription initiation start site for the necdin gene contains neuronrestricted promoter activity [16]. For the present study, this neuron-restrictive promoter region of the necdin gene was cloned from a mouse genomic DNA by PCR amplification of a $951 \mathrm{bp}$ of DNA fragment upstream of the translation initiation site $[17,18]$. The primers for necdin amplification were chosen from the mouse necdin sequence available from GenBank (accession number D76440). The forward primer corresponded to nucleotide numbers 1-26 (5'GATCATTTTC CACTAGAATC TTA ACG3') and the reverse primer corresponded to nucleotide numbers 956-937 (5'TCTGATCCGA AGGCGC AGAC3') [16]: a PCR reaction was done using Pwo DNA polymerase and the reaction was performed according to the manufacturer's guidelines (Roche Applied Science). Plasmid pUC19 was modified with restriction sites by sequential ligation of oligolinkers and a neomycin phosphotransferase (Neo) gene under the control of phosphoglycerate kinase promoter (PGK-1) was cloned. The presence of the Neo gene allows selection of stably transfected ES cells that confer resistance to antibiotic G418 [19]. To the Neo cloned plasmid vector the following DNA fragments were cloned sequentially: amplified DNA fragment (956 bp, promoter); the antibiotic resistance gene (919 bp, puromycin-N-acetyl-transferase gene or Pur'); internal ribosomal entry site (IRES); and the reporter gene ( $\beta$-galactosidase). The final plasmid vector with Necdin-Promoter-Selection-Reporter cassette was called pNec-SR vector (Figure 1).

\subsection{ES Cell Maintenance and Stable ES Cell Line Expressing Randomly Integrated Necdin Promoter-Selection-Reporter Cassette}

ES cells derived from 129/SvJ mice were maintained in culture on a layer of feeder cells consisting of mitomycin C-treated primary mouse embryonic fibroblasts (MEF) [20]. The ES cells were cultured in ES medium (Dulbecco's Modified Eagle Medium [DMEM]) supplemented

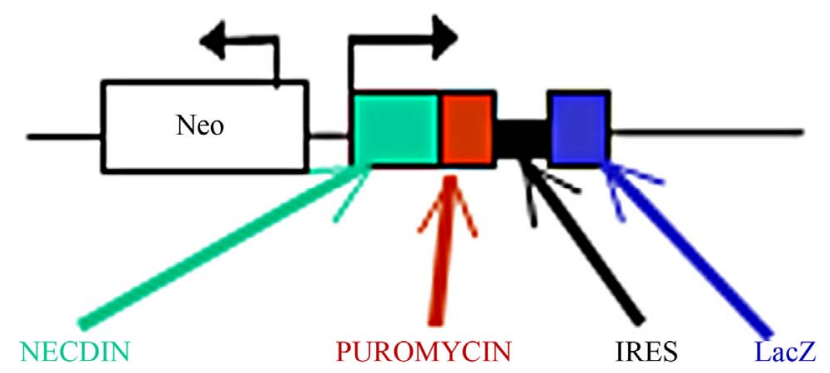

Figure 1. Targeting vector with Necdin promoter-selectionreporter (pNec-SR). Plasmid pUC19 was modified with restriction sites by sequential ligation of oligolinkers and the following gene fragments were sequentially cloned: a neomycin gene (Neo) under control of a phosphoglycerate kinase promoter (PGK); the amplified necdin promoter DNA fragment; the antibiotic resistance gene, puromycin-N-acetyl-transferase gene (Pur ${ }^{\mathrm{r}}$ ) IRES; and the reporter gene $\beta$-galactosidase.

with 15\% serum (Hyclone, Logan, UT), 1000 units $/ \mathrm{ml}$ LIF (ESGRO from Chemicon, Temecula, CA), $1 \mathrm{mM}$ sodium pyruvate, $1 \mathrm{mM}$ nonessential amino acids, 0.1 $\mathrm{mM}$ 2-mercaptoethanol, 25 units $/ \mathrm{ml}$ penicillin and 25 $\mu \mathrm{g} / \mathrm{ml}$ streptomycin. The ES cells were plated onto prepared feeder layers with freshly prepared ES medium and incubated in a humidified chamber $\left(37^{\circ} \mathrm{C}, 5 \% \mathrm{CO}_{2}\right)$. Media were changed every other day for maintenance of the ES cells.

A stable ES cell line with a randomly integrated necdin Promoter-Selection-Reporter cassette was made. Briefly, $10 \mu \mathrm{g}$ of pNec-SR vector was digested with 20 units of $\mathrm{Sfil}$ at $50^{\circ} \mathrm{C}$ for $2 \mathrm{hrs}$ (New England Biolabs). After digestion, the DNA was electrophoresed on agarose gel and purified using QIAEX II gel extraction kit (Qiagen). The linearized pNec-SR was electroporated ( $180 \mathrm{~V}, 500$ $\mu \mathrm{F})$ into ES cells and electroporated cells were plated onto tissue culture dishes. ES cells were cultured $\left(37^{\circ} \mathrm{C}\right.$, $5 \% \mathrm{CO}_{2}$ ) on an inactivated murine embryonic fibroblast (MEF) feeder layer in standard ES cell culture media containing LIF. After $24 \mathrm{~h}$, G418 selection was performed by addition of ES cell media containing G418 $(400 \mu \mathrm{g} / \mathrm{ml})$ and cells were allowed to grow for 5 days. Only cells transfected with plasmid survived in the presence of G418. The surviving colonies were picked, expanded and analyzed for the presence of $\beta$-galactosidase and puromycin genes by PCR analysis. Six colonies were positive: a single clone (ES-6) was selected as representtative for further studies. As a predifferentiation step, feeder cells were removed from the ES cell culture. To accomplish feeder cell removal, ES cells were passaged three times onto $0.1 \%$ gelatin-coated tissue culture plates using the following protocol. The cells were trypsinized (5 min) followed by trypsin inactivation (addition of an equal volume of media and incubation in a humidified chamber $\left.\left[15 \mathrm{~min}, 37^{\circ} \mathrm{C}, 5 \% \mathrm{CO}_{2}\right]\right)$. After incubation, most feeder cells remained attached to the plates whereas 
ES cells were in suspension. The ES suspension cells were transferred to $0.1 \%$ gelatin-coated tissue culture plates that supported ES cell growth and the presence/ absence of transferred feeder cells was microscopically checked. This process was repeated a second and a third time. After the third passage, all ES cells were completely devoid of feeder cells and were used for screening purposes.

\subsection{EB Formation and Test Compound Addition}

EBs were formed as described previously [7]. Single uniform sized EBs were formed in a multiwell plate, and each well contained a single embryoid body (EB) [7]. An ES-6 cell line containing a stably integrated necdinpuromycin-IRES- $\beta$-galactosidase cassette was allowed to form EB. Briefly, 1000 cells in $200 \mu \mathrm{l}$ of media without LIF were plated into 96-well PCR plates. On the third day, the EBs were transferred to laminin-coated 96-well tissue culture plates. The medium was changed on the fourth day and appropriate small molecule compounds were added with each compound being assessed in triplicate. Two positive controls, trans-retinoic acid (RA, at 1 $\mu \mathrm{M}$, Sigma-Aldrich), nerve growth factor (NGF, at10 $\mathrm{ng} / \mathrm{ml}$, Sigma-Aldrich), no treatment control, vehicle control (dimethylsufoxide: DMSO, Sigma Aldrich), and five small molecule compounds at $10 \mu \mathrm{M}$ concentration (piperine, curcumin, rosmarinic acid, U0126, LY294002; SigmaAldrich) were used as screening compounds for neuron differentiation. The cells were incubated for $48 \mathrm{~h}$ with compounds. After $48 \mathrm{~h}$, medium was removed, attached cells were rinsed with phosphate buffered saline (PBS) and medium was added. Cells were allowed to grow and differentiate in media for 4 days and lysed on the fifth day. Lysates were analyzed for $\beta$-galactosidase expression.

\subsection{Expression Analysis of Beta-Galactosidase and Neuronal Markers}

The level of neuron differentiation was measured using a sensitive chemiluminescent $\beta$-galactosidase assay (Clontech). Since activity of the necdin promoter conferred $\beta$-galactosidase expression, a quantitative measurement of $\beta$-galactosidase activity [21] was performed using cell lysate (cells exposed to compounds after day 5 of differentiation). Activity was normalized to total protein content of the cell lysates.

The neuronal expression of beta-galactosidase and neuronal markers was demonstrated by immunostaining. For in situ immunofluorescence, cells were grown on laminin-coated coverslips, rinsed with Tris-buffered saline and fixed with 4\% paraformaldehyde (in TBS, 10 min), washed with TBS and incubated with primary antibody (in $1 \%$ BSA, $05 \%$ Triton X100: overnight, $4^{\circ} \mathrm{C}$ ).
After primary antibody incubation, the cells were washed with TBS and incubated with fluorophore-conjugated secondary antibody in TBS containing Triton X100 (0.05\%) and $1 \%$ BSA (30 min). Cell types were visualized using fluorescence microscopy.

After incubation with screening compounds, the EBs were dissociated by gently pipetting and plated on laminincoated coverslips. On day 12 , the cells were fixed and double-immunostained with low-affinity nerve growth factor receptor (LNGFR) also called p75 (p75, murine monoclonal antibody; Sigma-Aldrich) and beta-galactosidase (rabbit polyconal antibody; abcam) followed by flurophore-conjugated secondary antibody (Alexa-568 conjugated to anti-mouse, Alexa-488 to goat anti-rabbit respectively; Molecular Probes). Cells were stained with 4',6-diamidino-2-phenylindole (DAPI; Molecular Probes) to display cell nuclei.

\subsection{Enrichment for Neurons Using Selection}

Retinoic acid-differentiated cells were used to demonstrate that the selection method enriched for neurons. EBs were exposed to RA for $48 \mathrm{~h}$ and then the compound was removed. Cells were allowed to grow on laminin-coated coverslips for immunostaining or in 96 well microplate for measurement of beta-galctosidase expression. After retinoic acid exposure, cells were divided into two populations and changed to cell culture media. Two days after growing in regular media, cells were subjected to puromycin selection. One group was exposed to puromycin $(+)$ and another group, the no selection group, had no puromycin (-). After $12 \mathrm{~d}$, cells were lysed and betagalactosidase activity was measured. Similar experiments were carried out on laminin-coated coverslips for immunocytochemistry. Cells were stained for the neuron marker, microtubule associated protein 2 (MAP2), and the astrocyte marker, glial fibrilary acidic protein (GFAP). Fluorescence stained cells were counted microscopically in at least three fields.

\section{RESULTS}

\subsection{Neuron-Restricted DNA Fragment of Necdin Promoter Expresses Beta-Galctosidase Activity in Neurons}

Stably transfected ES cells containing the Necdin Promoter-Selection-Reporter vector (pNec-SR vector, Figure 1) were differentiated into neuronal cells by retinoic acid addition. To verify that necdin promoter drove the beta-galactosidase reporter in neuronal cells, the retinoic acid-differentiated cells grown on laminin-coated coverslips on day 12 were fixed and immunostained for the neuronal marker, low-affinity nerve growth receptor (LNGFR) and for beta-galactosidase (Figure 2(A)). As expected, cells expressing LNGFR also expressed beta- 
galactosidase (Figure 2(A)) indicating that the necdin promoter expressed beta-galactosidase in neurons. The same area of Figure 2(A) is shown in bright field microscopy in Figure 2(B), and the nuclei, visualized by DAPI stain, of the cells are shown in Figure 2(C).

\section{2. $\beta$-Galactosidase Activity as an Indicator of Post-Mitotically Differentiated Neurons}

The level of neuron differentiation was measured using a sensitive chemiluminescent $\beta$-galactosidase assay (Clontech). Since activity of the necdin promoter conferred $\beta$-galactosidase expression, a quantitative measurement of $\beta$-galactosidase activity was performed using cell lysate. Compounds were removed after $48 \mathrm{~h}$ and cells were allowed to differentiate for $4 \mathrm{~d}$ in laminin-coated 96 well microplates. Activity was normalized to the total protein content of the cell lysates. Two positive controls, NGF and RA, and five small molecule compounds (piperine, curcumin, rosmarinic acid, U0126, LY294002) were used for screening (Figure 3). Fold increase was calculated based on vehicle control ( $\beta$-galactosidase activity). NGF showed more neurogenesis (30-fold) than did RA (11fold), a result that was not surprising since NGF is a stronger neurogenic growth factor than RA. Piperine had a 3.3-fold increase in neurogenesis that was significantly different from the positive controls. In this select set of 5 test compounds, the positive controls (RA and NGF) demonstrated that the differentiated neurons are measurable by beta-galactosidase reporter expression. However, since none of the 5 test compounds displayed results equal to or greater than the controls (NGF or RA) and since this had been determined to be the screening strategy, the interpretation of this EB-based screen was that none of these test compounds had neurogenesis potential (Figure 3).

\subsection{Selection Marker Enriches for Neurons}

Exposure of differentiated cells to puromycin should have allowed the survival of neurons because only neurons expressed puromycin under control of the necdin promoter. Cells were lysed on the 12th day with or without

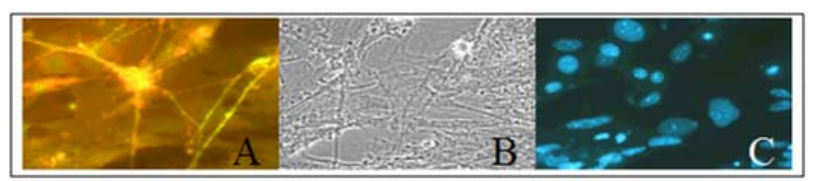

Figure 2. The necdin promoter expresses beta-galactosidase in neurons only (day 12 after selection). (A) Double immunostaining using primary $\mathrm{Ab}$ for beta-galactosidase (murine $\mathrm{mAb}$ ) and low affinity NGF (p75) receptor (rabbit pAb) followed by fluorophore-conjugated secondary Ab (Alexa-568 conjugated goat anti-mouse; Alexa-488 goat anti-rabbit, respectively); (B) Same area as A viewed with bright field microscopy; (C) Same area as A stained with DAPI to display cell nuclei.

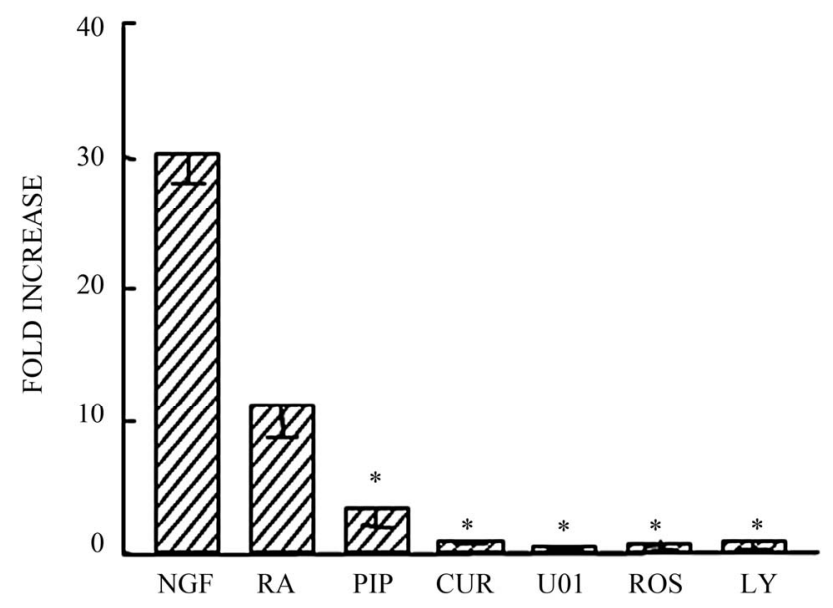

Figure 3. Effects of compounds on cell differentiation. Embryoid bodies were screened with controls and small molecule compounds ( $\mathrm{n}=5$ for NGF: $\mathrm{n}=7$ for RA with piperine and $\mathrm{n}=$ 3 for others). Fold increase was calculated based upon vehicle alone treatment of control. Small molecule results indicated significant $(\mathrm{p}<0.05)$ differences of all agents compared with NGF and RA. NGF $=$ nerve growth factor. $\mathrm{RA}=$ retinoic acid. $\mathrm{PIP}=$ piperine. $\mathrm{CUR}=$ curcumin. $\mathrm{U} 01=\mathrm{U} 0126 . \mathrm{ROS}=$ rosmarinic acid. $\mathrm{LY}=\mathrm{LY} 294002$. Results are mean \pm SEM. ${ }^{*} \mathrm{p}<0.05$.

puromycin treatment and the beta-galactosidase activity was measured. As shown in Figure 4(A), a puromycintreated population exhibited greater beta-galactosidase activity than an untreated population. Next, cells were immunostained and the cells displaying marker expression (MAP2 for neurons and GFAP for astrocytes) were counted. Percent positive cells based on neuron and astrocyte markers, MAP2 and GFAP, were evaluated in the puromycin-treated verus untreated differentiated neuronal cells. As shown in Figure 4(B), puromycin selection eliminated astrocytes but enriched for neurons.

\section{DISCUSSION}

The ability to differentiate ES cells in vitro allows for their use as model systems for the study of developmental potential and also as valuable reagents for stem cell therapeutic approaches [22-24]. The method proposed here is an efficient in vitro system to screen and study effects of small molecules and bioagents. A positive hit criterion that identifies a compound as one exhibiting differentiation potential is its having similar or higher reporter expression level than that of positive controls. In this present study, positive controls were retinoic acid and nerve growth factor. None of the five compounds used for neuronal differentiation produced reporter expression similar to or greater than the positive controls. If compounds are identified that have equal or greater reporter expression than positive controls in a larger screening strategy, then these compounds can be further evaluated for differentiation potential. Using appropriate cul- 

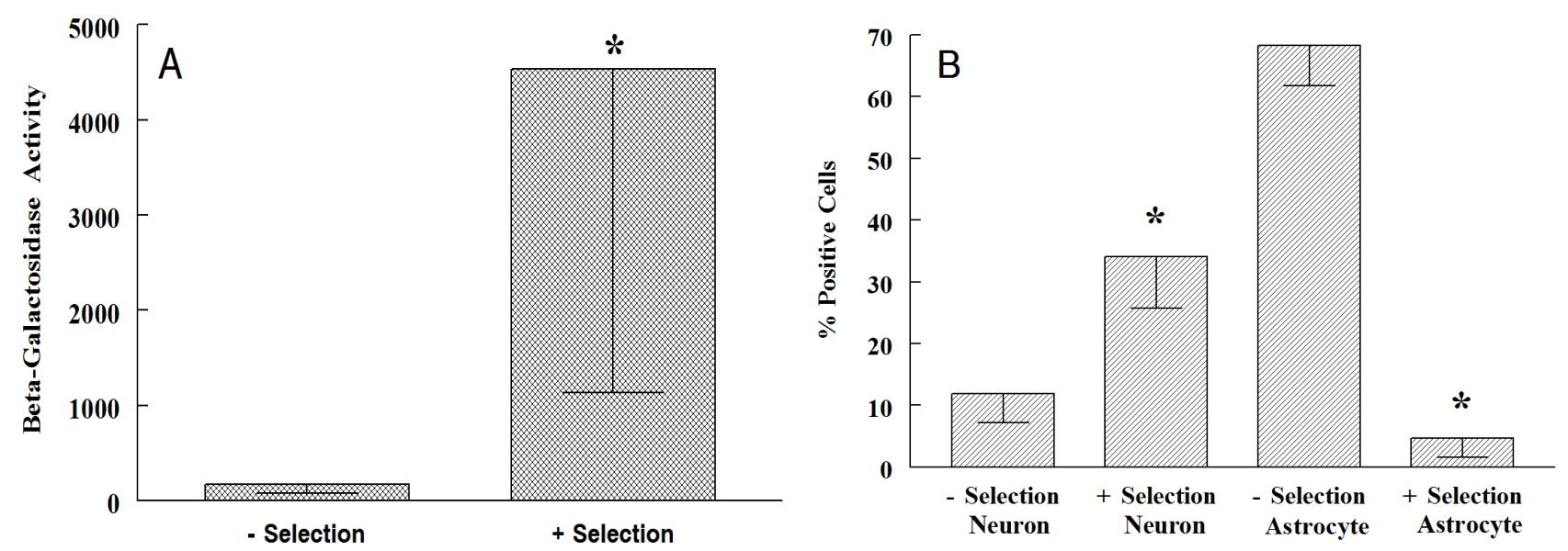

Figure 4. Puromycin selection (day 12). (A) Beta-galactosidase activity measured in cells treated without (- selection) and with $(+$ selection) puromycin. A significant difference $(\mathrm{p}<0.05)$ between selection $(+)$ and no selection $(-$ selection $)$ was noted in cells with beta-galactosidase activity (results are mean \pm SEM. $n=3$ ); (B) Cells were stained with markers for neurons (MAP2 a $+b)$ and astrocytes (GFAP) and then number of cells was counted. At least 3 areas on each slide were counted. A significant $(\mathrm{p}<0.05)$ difference was observed between selected and unselected neurons and between selected and unselected astrocytes. Results reported as mean \pm SEM. $\mathrm{n}=3 .{ }^{*} \mathrm{p}<0.05$.

ture conditions, ES cells differentiate and form EBs that contain cells of hematopoietic, endothelial, muscular and neuronal lineages [25-29]. Many aspects of lineage-specific differentiation programs observed within EBs reflect those found in the embryo indicating this model system provides access to early cell populations that develop in a normal fashion [2,30]. ES cells are also able to spontaneously differentiate and generate various lineages under appropriate conditions in cell culture [31,32]. Using this in vitro system, it is possible to screen many compounds efficiently and, once a candidate molecule is identified, it can be studied in detail to define mechanisms by which it exerts its effect. For screening purposes, uniform-sized EBs have been shown to have synchronous differentiation potentials [6]. The reason for generating uniform-sized EBs is that factors produced by cells present in EBs may influence the differentiation pathway; therefore, it is critical to have uniform-size EBs for screening purposes $[23,28]$.

Effects of small molecules may be studied using neurons formed by an EB differentiation method or by direct differentiation from ES cells with no EB intermediate step. Comparison studies of developmental patterns of murine EBs versus in vivo murine embryos have been performed using gene expression and tagged reporter constructs [2]. Results indicate that prior to day 3, EBs in suspension culture are equivalent developmentally to pregastrulation-stage embryos (4.5 - 6.5 days post coitum [d.p.c]). Between days 3 and 5, EBs contain cell types present in embryos during gastrulation (6.5 - 7.5 d.p.c.). After day 6, EBs are equivalent to embryos in the stage of early organogenesis (7.5 d.p.c) [2]. The EB differentiation method is superior, however, because the EB neuro- genesis process proceeds in a manner similar to how it actually occurs in a developing embryo. As an example, the drug thalidomide has anti-angiogenic effects in vivo; in other words, it inhibits endothelial cell formation. This anti-angiogenic effect was not observed in either an endothelial cell culture system or a chorioallantoic membrane assay; however, anti-angiogenesis was noted in an EB-based assay [33]. Finding the anti-angiogenic effect of thalidomide only in an EB culture system and not by other screening methods strongly indicates the need to recapitulate intact organism development in small molecule screens.

The method described in this paper allows identification of differentiated cells by reporter gene expression and selective enrichment of that particular cell population. The differentiated cell type is then identified by analysis of reporter gene expression. The importance of the selection marker is selective enrichment of that particular cell population. By creation of a panel of lineagespecific promoter-reporter ES cell lines, screening of EBs from these cell lines will provide an excellent system for testing differentiation effects of small molecules into many different cell lineages.

\section{ACKNOWLEDGEMENTS}

The author sincerely thank the critical evaluation of the manuscript by Rita M. Heuertz and Sally Triocmi. This study is supported by a grant from NIH/NINDS (1R43NS046133).

\section{REFERENCES}

[1] Xian, H.Q. and Gottlieb, D.I. (2001) Peering into early neurogenesis with embryonic stem cells. Trends in Neu- 
rosciences, $\mathbf{2 4}, 685-686$. doi:10.1016/S0166-2236(00)01966-4

[2] Leahy, A., Xiong, J.W., Kuhnert, F. and Stuhlmann, H. (1999) Use of developmental marker genes to define temporal and spatial patterns of differentiation during embryoid body formation. Journal of Experimental Zoology, 284, 67-81. doi:10.1002/(SICI)1097-010X(19990615)284:1<67::AID -JEZ10>3.0.CO;2-O

[3] Bain, G. and Gottlieb, D.I. (1998) Neural cells derived by in vitro differentiation of P19 and embryonic stem cells. Perspectives on Developmental Neurobiology, 5, 175178.

[4] Okabe, S., Forsberg-Nilsson, K., Spiro, A.C., Segal, M. and McKay, R.D. (1996) Development of neuronal precursor cells and functional postmitotic neurons from embryonic stem cells in vitro. Mechanisms of Development, 59, 89-102. doi:10.1016/0925-4773(96)00572-2

[5] Wichterle, H. and Peljto, M. (2008) Differentiation of mouse embryonic stem cells to spinal motor neurons. Current Protocols in Stem Cell Biology, Chapter 1: Unit-1H.

[6] Ng, E.S., Davis, R.P., Azzola, L., Stanley, E.G. and Elefanty, A.G. (2005) Forced aggregation of defined numbers of human embryonic stem cells into embryoid bodies fosters robust, reproducible hematopoietic differentiation. Blood, 106, 1601-1603. doi:10.1182/blood-2005-03-0987

[7] Ezekiel, U.R., Muthuchamy, M., Ryerse, J.S. and Heuertz, R. (2006) Single embryoid body formation in a multiwell plate. Electronic Journal of Biotechnology, 10, 328335.

[8] Bain, G., Ray, W.J., Yao, M. and Gottlieb, D.I. (1996) Retinoic acid promotes neural and represses mesodermal gene expression in mouse embryonic stem cells in culture. Biochemical and Biophysical Research Communications, 223, 691-694. doi:10.1006/bbrc.1996.0957

[9] Chaerkady, R., Kerr, C.L., Marimuthu, A., Kelkar, D.S., Kashyap, M.K., Gucek, M., Gearhart, J.D. and Pandey, A. (2009) Temporal analysis of neural differentiation using quantitative proteomics. Journal of Proteome Research, $\mathbf{8}$, 1315-1326. doi: $10.1021 / \mathrm{pr} 8006667$

[10] Miloso, M., Bertelli, A.A., Nicolini, G. and Tredici, G. (1999) Resveratrol-induced activation of the mitogen-activated protein kinases, ERK1 and ERK2, in human neuronblastoma SH-SY5Y cells. Neuroscience Letters, 264, 141-144. doi:10.1016/S0304-3940(99)00194-9

[11] Ahlemeyer, B. and Krieglstein, J. (2003) Neuroprotective effects of Ginkgo biloba extract. Cellular and Molecular Life Sciences, 60, 1779-1792. doi:10.1007/s00018-003-3080-1

[12] Yoo, D.Y., Nam, Y., Kim, W., Yoo, K.Y., Park, J., Lee, C.H., Choi, J.H., Yoon, Y.S., Kim, D.W., Won, M.H., et al. (2011) Effects of Ginkgo biloba extract on promotion of neurogenesis in the hippocampal dentate gyrus in C57BL/6 mice. Journal of Veterinary Medical Science, 73, 71-76. doi:10.1292/jvms.10-0294

[13] Mahonen, A., Jaaskelainen, T. and Maenpaa, P.H. (1996) A novel vitamin D analog with two double bonds in its side chain. A potent inducer of osteoblastic cell differen- tiation. Biochemical Pharmacology, 51, 887-892. doi:10.1016/0006-2952(95)02242-2

[14] Watanabe, K., Ueno, M., Kamiya, D., Nishiyama, A., Matsumura, M., Wataya, T., Takahashi, J.B., Nishikawa, S., Nishikawa, S., Muguruma, K., et al. (2007) A ROCK inhibitor permits survival of dissociated human embryonic stem cells. Nature Biotechnology, 25, 681-686. doi: $10.1038 / \mathrm{nbt} 1310$

[15] Li, Z., Theus, M.H. and Wei, L. (2006) Role of ERK 1/2 signaling in neuronal differentiation of cultured embryonic stem cells. Development, Growth \& Differentiation, 48, 513-523. doi:10.1111/j.1440-169X.2006.00889.X

[16] Uetsuki, T., Takagi, K., Sugiura, H., and Yoshikawa, K. (1996) Structure and expression of the mouse necdin gene. Identification of a postmitotic neuron-restrictive core promoter. Journal of Biological Chemistry, 271, 918-924. doi:10.1074/jbc.271.2.918

[17] Kuo, C.H., Uetsuki, T., Kim, C.H., Tanaka, H., Li, B.S., Taira, E., Higuchi, H., Okamoto, H., Yoshikawa, K. and Miki, N. (1995) Determination of a necdin cis-acting element required for neuron specific expression by using zebra fish. Biochemical and Biophysical Research Communications, 211, 438-446. doi:10.1006/bbrc.1995.1833

[18] Maruyama, E. (1996) Biochemical characterization of mouse brain necdin. Biochemical Journal, 314, 895-901.

[19] Tucker, K.L., Wang, Y., Dausman, J. and Jaenisch, R. (1997) A transgenic mouse strain expressing four drugselectable marker genes. Nucleic Acids Research, 25, 3745-3746. doi:10.1093/nar/25.18.3745

[20] Brook, F.A. and Gardner, R.L. (1997) The origin and efficient derivation of embryonic stem cells in the mouse. Proceedings of the National Academy of Sciences of the United States of America, 94, 5709-5712. doi:10.1073/pnas.94.11.5709

[21] Martin, C.S., Wight, P.A., Dobretsova, A. and Bronstein, I. (1996) Dual luminescence-based reporter gene assay for luciferase and beta-galactosidase. Biotechniques, 21 520-524.

[22] Xiong, J.W., Battaglino, R., Leahy, A. and Stuhlmann, H. (1998) Large-scale screening for developmental genes in embryonic stem cells and embryoid bodies using retroviral entrapment vectors. Developmental Dynamics, 212, 181-197. doi:10.1002/(SICI)1097-0177(199806)212:2<181::AIDAJA4>3.0.CO;2-D

[23] Laposa, R. (2011) Stem cells for drug screening. Journal of Cardiovascular Pharmacology, 58, 240-245. doi:10.1097/FJC.0b013e31821823f5

[24] Barbaric, I., Jones, M., Harley, D.J., Gokhale, P.J. and Andrews, P.W. (2011) High-content screening for chemical modulators of embryonal carcinoma cell differentiation and survival. Journal of Biomolecular Screening, 16 603-617. doi:10.1177/1087057111406547

[25] Schmitt, R.M., Bruyns, E. and Snodgrass, H.R. (1991) Hematopoietic development of embryonic stem cells in vitro: Cytokine and receptor gene expression. Genes \& Development, 5, 728-740. doi:10.1101/gad.5.5.728

[26] Risau, W., Sariola, H., Zerwes, H.G., Sasse, J., Ekblom, P., Kemler, R. and Doetschman, T. (1988) Vasculogene- 
sis and angiogenesis in embryonic-stem-cell-derived embryoid bodies. Development, 102, 471-478.

[27] Wang, R., Clark, R. and Bautch, V.L. (1992) Embryonic stem cell-derived cystic embryoid bodies form vascular channels: An in vitro model of blood vessel development. Development, 114, 303-316.

[28] O'Shea, K.S. (1999) Embryonic stem cell models of development. Anatomical Record, 257, 32-41. doi:10.1002/(SICI)1097-0185(19990215)257:1<32::AIDAR6>3.0.CO;2-2

[29] Kane, N.M., Xiao, Q., Baker, A.H., Luo, Z., Xu, Q. and Emanueli, C. (2011) Pluripotent stem cell differentiation into vascular cells: A novel technology with promises for vascular re(generation). Pharmacology \& Therapeutics, 129, 29-49. doi:10.1016/j.pharmthera.2010.10.004

[30] Vallier, L., Mancip, J., Markossian, S., Lukaszewicz, A., Dehay, C., Metzger, D., Chambon, P., Samarut, J. and Savatier, P. (2001) An efficient system for conditional gene expression in embryonic stem cells and in their in vitro and in vivo differentiated derivatives. Proceedings of the National Academy of Sciences of the United States of America, 98, 2467-2472. doi:10.1073/pnas.041617198

[31] Doss, M.X., Koehler, C.I., Gissel, C., Hescheler, J. and Sachinidis, A. (2004) Embryonic stem cells: A promising tool for cell replacement therapy. Journal of Cellular and Molecular Medicine, 8, 465-473. doi:10.1111/j.1582-4934.2004.tb00471.x

[32] Odorico, J.S., Kaufman, D.S. and Thomson, J.A. (2001) Multilineage differentiation from human embryonic stem cell lines. Stem Cells, 19, 193-204. doi:10.1634/stemcells.19-3-193

[33] Sauer, H., Gunther, J., Hescheler, J., and Wartenberg, M. (2000) Thalidomide inhibits angiogenesis in embryoid bodies by the generation of hydroxyl radicals. American Journal of Pathology, 156, 151-158. doi:10.1016/S0002-9440(10)64714-1 